\title{
A Pricing Policy to Mitigate Interference Management in Femtocell Network
}

\author{
O. A. Akinlabi, B. S. Paul, M. Joseph, and H. C. Ferreira
}

\begin{abstract}
Recently, the concepts of game theory has attract the attention of researcher for self-governing decision making to study the performance of wireless network. There has been growing interest in accepting game theoretic method to wireless systems. The problem of interference management in femtocell network is approached from the prospective of game theory with the concept of price policy. Whereby price is used to control the power transmission of each femto users in the cell coverage. In this paper, we propose a price policy to reduce the effect of interference between the femto users at both center and edge regions of cell network. The performance of our proposed algorithm is demonstrated by simulation results.
\end{abstract}

Index Term-Femtocells, nash equilibrium, OFDDMA system, interference.

\section{INTRODUCTION}

The key feature of Orthogonal Frequency Division Multiple Access (OFDMA) systems is that it copes with severe channel condition without complex equalization fit [1], which can meet a variety of service requirements in the network systems. The access point of femtocell, which is deployed as Home evolved Node-B (HeNB) in 3GPP, are for connectivity of broadband to the operator core network, for cognitive facilities. Femtocell are aimed with cognitive functionalities for loading, capacity and coverage optimization, random access channel handover parameter and self- optimization to maintain quality of service with minimal intervention from the Mobile Operators [2]. With this motivation mentioned, this paper considers interference management in femtocell network, which consists of centralized Base Station and femtocell base stations. By integrating the cognitive capabilities into centralized approach under fractional frequency reuse and aiming to minimize the utility by using the unoccupied frequencies while minimizing interference to the primary base station in a spectrum overlay. The formulation of interference management in femtocell network with game-theoretic setting and using the Nash equilibrium as the balance solutions of the formulated problem. Game-theoretic approaches to power control [3]-[6] describe Quality of service (QoS) for the mobile station using utility functions. The idea of utility function is generally used in microeconomics and game theory to denote the level of

Manuscript received February 6, 2014; revised May 20, 2014. This work was supported in part by the University of Johannesburg.

A. O. Akinlabi, B. S. Paul, M. Joseph, and H. C. Ferreira are with the Electrical and Electronic Engineering Technology Department, University of Johannesburg, Johannesburg, South Africa, SA (e-mail: akinlabiakindeji@gmail.com). agreement of a decision-maker with specific products or services as a result of its actions. Game theory is used to model interactions among self-interested users and predict their choice of strategies to optimize utility functions until a Nash equilibrium is reached, where no user can further increase its corresponding utility through individual action. Utility functions used for wireless systems relay on SIR and transmitted power of a given station. In [7], the authors provide some incentive for using game theory approach to study wireless network systems, and in particular power control. In [8] and [9], power control is modelled as a non-cooperative game in which users choose their transmit powers in order to maximize their utilities, where utility is defined as the ratio of throughput to transmit power .

It's more preferable to use the Nash equilibrium as the solution since it directly considers the ability of the players to coordinate their actions, which can lead to higher performance if each player behaves to act in selfish nature One of the main focuses is how to mitigate interference from the multiple femtocell users at both regions of the cell coverage network so as to improve the system performance and quality of service in the networks.

The model proposed in this paper is based on a simple game theoretical relationship between the base station at the center of the cell site coverage and femtocell base station with the concepts of price policy.

The objective of this model is to monitors and fulfils the following aims;

- The usage patterns of users that administrate the necessary requirements for the quality of performance in the network.

- The policy enhances the use of the common resources for reliable transmission.

- The policy promotes fairness and quality of service among the network users.

The remaining of this paper is structured as follows. Interference management in femtocell network is explained in Section II. Section III describes the theoretical approach of the system. Section IV analyses interference management with price policy and Section $\mathrm{V}$ explain interference management without price policy. The performance evaluation are shown in Section VI. Finally, we conclude our paper in Section VII.

\section{INTERFERENCE MANAGEMENT IN FEMTOCELL NETWORK}

In this section, a base station is located at the Centre of heterogeneous cells with a number of femtocells randomly deployed by the home and offices users of the network. By integrating the cognitive capacities of femtocell base station, 
hence the base station occupied at the center is known as the primary base station and that of femtocell, known as the secondary base station in a spectrum overlay network. Due to their self-behavior of each users of femtocell at each region of the cell coverage, the competition for the common license spectrum resource among the cognitive base stations can be formulated using a game-theoretic concept. The Fig. 1 depicts a centralized base station with a number of femtocell base station randomly deployed with in an OFDMA network. This type of a network will be used in the simulations.

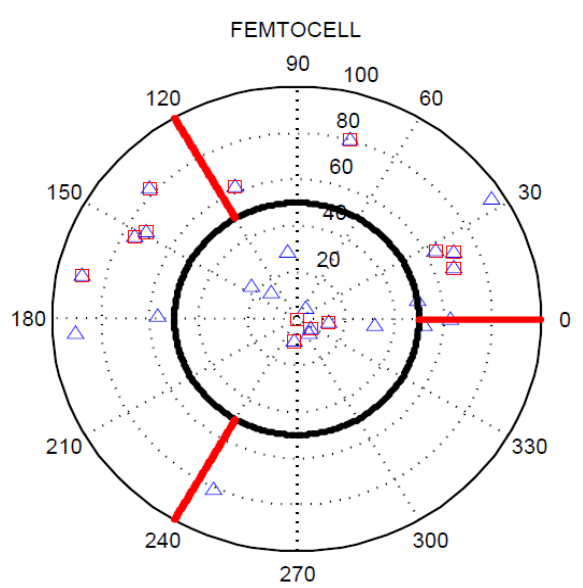

Fig. 1. A centralized base station with multiple femtocell base station.

\section{SYSTEM DESCRIPTION}

The interference management in femtocell can be modeled using game theoretically approach with the concepts of price mechanism. In this paper, femtocell users is denoted as $k=$ $(1,2 \ldots J)$ who want to share the common license band with the primary base station. The price policy will enhance the usage of the common license band without interference between the femtocell users at the center and edge of the cell coverage.

The total available band divided into sub channel denoted by $\Delta f$. The transmission power of their serving femtocell and neighboring femtocell $F^{i}$ on subcarrier $i$, respectively are denote by $P_{f, i}$ and $P_{F^{i}, i}$ the channel gain between the transmitting femtocell user $k$ and the serving femtocell on subcarrier $i$, were indicate with $G_{f, F, i}$ while $G_{f, F^{i}, i}$ corresponds to channel gain from the interfering neighboring femtocell $F^{i} . P_{M, i}$ indicates the transmitting power of the neighboring macrocell $M$ on subcarrier $i$. $G_{f, M, i}$ denote channel gain between femto user $k$ and the neighboring macrocell $M$ on subcarrier $i$. The utility function is denoted as $u_{i}$, and the set of transmit power is represents as $p_{i}=\left\{p_{i}: p_{i} \in\left[0, p_{\max }\right]\right\}$. Finally, $N_{0}$ is the White Noise Power. The analysis of the performance was carried out in terms of signal to interference noise ratio (SINR) of both the regions of the cell coverage, and the utility function. The received SINR for the femtocell user $k^{\text {th }}$ located at the cell center region on a subcarrier $i$ can be expressed as [10]

$$
\gamma=\frac{P_{f, k} G_{f, F, k}}{N_{0}+\sum_{M} P_{M, k} G_{f, M, k}+\sum_{F^{i}} P_{F^{i}, k} G_{f, F^{i}, k}} \forall_{f, k}\{1,2, \ldots J\}
$$

where, $F^{i}$ is the set of interfering femtocell.

The path loss between a Femtocell user base station and $\mathrm{UE}$ in the same house is given by the following equation [10]

$$
P L_{f, i}(d B)=38.46+20 \log 10(d(m))+L_{\text {walls }}
$$

where $L_{\text {walls }}$ is 7,10 and $15 d B$ for light internal, internal and external walls respectively [11], so the channel gain is written as [10]

$$
G=10^{-P L / 10}
$$

The capacity of the cell center is estimate in bits/s of a wireless system is expressed as [11]

$$
C_{i, c e n t r e}=\Delta f \cdot\left[\log 2\left(1+\alpha \gamma_{f, k}\right) \quad k=1, \ldots J\right.
$$

The capacity of cell edge user is estimate in bits/s of a wireless system is expressed as [11]

$$
C_{i, e d g e}=\Delta f \cdot\left[\log 2\left(1+\alpha \gamma_{f, k}\right) \quad k=1, \ldots J\right.
$$

Finally, the total network capacity is expressed in (bits/s);

$$
C_{k, \text { Total }}=C_{k, \text { centre }}+C_{k, \text { edge }}
$$

where $\alpha=\frac{-1.5}{\operatorname{In}(B E R)}$

Here we set $B E R$ to $10^{-6}$.

Power has been one the resources used to suppress interference in the wireless system, by imposing cost on the power transmission whereby the signal of the femtocell user which are closer to the base station at the cell center will be controlled and it will not cause any unwanted interference to the entire network. We define our interference received at the user side mathematically;

$$
I=N_{0}+\sum_{M} P_{M, k} G_{f, M, k}+\sum_{F^{i}} P_{F^{i}} G_{f, F^{i}, k} \forall_{f, k}\{1, \ldots j\}
$$

From the SINR, we can formulate our power as;

$$
p=\frac{\gamma_{f, k} I_{f, k}}{G_{f, k}}
$$

\section{INTERFERENCE MANAGEMENT WITH PRICE POLICY}

The main aim of the section is to improve the signal levels of femtocells so as to avoid interference under fractional frequency re-use. Generally, this section aims to show how pricing within the femtocells can influence user's behavior so that all users reach a Nash equilibrium state. This subsection defines a utility function to evaluate the overall system performance, based on SINR of all the users in the network. It 
is preferable to model the user's utility as concave increasing function of the user's SINR [12], [13]. The best way for a user to maximize is utility function in a non-cooperative game, when transmitting with highest possible power, such that the level of interference in the network is increased and thus reducing the whole spectral efficiency rather than improving it.

In order to control this, it is necessary to impose a price term (or cost) so that it prevents the users from always transmitting at full power. We aim to model utility functions which ensure optimal SINR so as to provide the best quality of service amongst the contending users of the base station.

Mathematically the utility function can be expressed as in equation, for both center and edge region of the cell coverage in the equation below [11]

$$
\begin{aligned}
& u_{k, \text { centre }}=\beta \log 2(1+\alpha \gamma)-c_{k}\left(p_{k}\right) \\
& u_{k, \text { edge }}=\beta \log 2(1+\alpha \gamma)-c_{k}\left(p_{k}\right)
\end{aligned}
$$

where $c_{k}$ is the price that determine the cost of power transmission, the user at the edge zone to transmit at the higher power due to their channel weakness and also to overcome the higher interference from the user at the cell center zone, the price for the zone should relatively low which promote fairness to the user at the edge zone. However, the user at the center zone experience strong channel and weak interference because they are closer to the base station. Therefore they should be restricted to transmit at the higher power so that they will not cause unnecessary interference to the whole system. The price must be relatively higher in value.

In the problem above, the problem is to find the Nash equilibrium point from which both regions have incentive to deviate.

Definition 1: the pure strategy solution for Nash Equilibrium (NE) for the proposed problem is when $u_{i, \text { centre }}=u_{i, \text { edge }}$ constitutes the solution for the problem for the user at both regions, and then the point of NE is met. Hence, the Nash equilibrium is the solution of the game.

Proof: it is notice here that the quality of service was improved and Nash equilibrium served has the solution to the interference in femtocell network.

\section{Interference Management without Price Policy}

This section provides a game without price intervention, where the Base Station (BS) at the center is engaged with multiple femtocell users from both regions for the purpose of transmission. The femtocell users act independently and care only about themselves, they take into account each other's potential decision, and the band used. This leads to interference and poor quality of service to network users, especially user that are far from the base station would suffer most. In this model, every user's basic utility will be maximized, if a zero-price is applied to the user who want to access and share the frequency band (i.e. $p_{i}=0$ ) without any price term, then every femto user will transmit at the high power for their transmission of data in respect of your region. We observed that each user is selfish and will utilize the frequency band without restraint, and the whole network user will try to maximize their usage so as to yield a maximum positive payoff.

In a non- price intervention policy, user who want to share the license band will try to maximize its own utility. Thus, the utility function in game without price intervention is given as

$$
\begin{gathered}
u_{k, \text { centre }}=\beta \log 2(1+\alpha \gamma) \forall k \in\{1 \ldots . . j\} \\
u_{k, \text { edge }}=\beta \log 2(1+\alpha \gamma) \forall k \in\{1 \ldots . j\}
\end{gathered}
$$

Theorem 1: The existence of NE at which is the solution to the problem at both region for the usage of frequency band at which the power of each user is set to its maximum value. This NE reduces the quality and performance of network.

Proof: it can be observed here that each user of the cell network coverage will transmit at the high power in order to transmit their data to the base station and more so they will be dependent on themselves to improve the quality of service. The NE will cause too much interference and reduces quality performance of the whole networks for all users.

Proposed algorithm for formulated problem: interference management in femtocell network.

The proposed algorithm was used to simulate the formulated problem: interference management in femtocell network. The algorithm was run in MATLAB, where by resulting to Nash equilibrium point between the center and edge regions of the cell networks for the solutions.

Generate_cell \% centre and edge region

Generate_base station \% at the cell centre region

Drop femto_user at both regions

Calculate SINR at the centre region

Calculate SINR at the edge region

Measure the utility function of centre region

if $U_{\text {Centre }}>U_{\text {edge }}$

update the price for the centre region i.e. $p+1, p+2, . . P$

else if $U_{\text {Centre }}<U_{\text {edge }}$

update the price for the edge region

else if $U_{\text {Centre }}=U_{\text {edge }}$

stop

Nash equilibrium find

end if

end

\section{PERFormance EVAluation}

The simulation parameter that will be used for experiment are listed in Table I. The simulation analysis is basis on the mathematical implementation for performance evaluation and, however, the utility function of the femtocell user at the various regions of the cell coverage. The positions of femtocell users in the cell coverage area, and as well as other parameters enable us to calculate for transmitting power of the regions and signal, as well as interfere to which it affect the networks of the cell coverage. The price intervention to the power transmission to each users help to mitigate the interference in which that users would not transmit at the high power, especial those closer to the base station. Based on this 
estimation, we consider the White Additive Gaussian noise, to be able to make an assessment of the SINR and also the utility function.

In this section, we present the numerical results and analysis to demonstrate the network performance of the system and the proposed algorithm. However, the bandwidth operates in $30 \mathrm{MHz}$ band. The radius of femtocell user at the center region form the base station is $50 \mathrm{~m}$, while the radius of femtocell user at cell edge region from the base station is $100 \mathrm{~m}$.

The total bandwidth is denoted as $\left(N_{b a n d}\right)$, which is divided into sub-channel with amplitude of $15 \mathrm{kHz}$. The transmission powers of each region are differ in respect to their location. Transmission power for femto user at the center region is $P_{\text {max,centre }} 10 \mathrm{dBm}$ and transmission power for femto user at cell edge region is $P_{\text {max,edge }} 25 \mathrm{dBm}$. This allowed in order, to compensate their channel weakness and also to over the higher interference from the user at the center region.

The white Additive Gaussian Noise of femto user at both regions is figures to be $-174 \mathrm{dBm} / \mathrm{Hz}$. The price policy interference management is to promote fairness between the two regions where the femtocell user operate, whereby maximizing the power transmission through their utility function.

TABLE I: SIMULATION PARAMETER

\begin{tabular}{|l|l|}
\hline Cell Parameters & \\
\hline Centre radius & $50 \mathrm{M}$ \\
\hline Edge radius & $100 \mathrm{M}$ \\
\hline Number of users & 40 \\
\hline Total bandwidth & $30 \mathrm{MHz}$ \\
\hline Sub-carrier spacing & $15 \mathrm{~Hz}$ \\
\hline Path loss & $38.46+20 \log 10(\mathrm{~d}(\mathrm{~m}))+\mathrm{L}_{\text {walls }}$ \\
\hline Transmission power at edge & $25 \mathrm{~dB}$ \\
\hline Transmission power at center & $10 \mathrm{~dB}$ \\
\hline
\end{tabular}

The main objective of our simulation was to find a point of Nash equilibrium of the utility function of each femto user at each regions of the cellular networks, where interference is mitigate and improve the network performance for both regions of the femtocell users. In order to mitigate interference, we simulate our proposed algorithm for formulated problem. The point of Nash equilibrium of each regions reduce the presence of interference. The results are portrayed in the following figures.

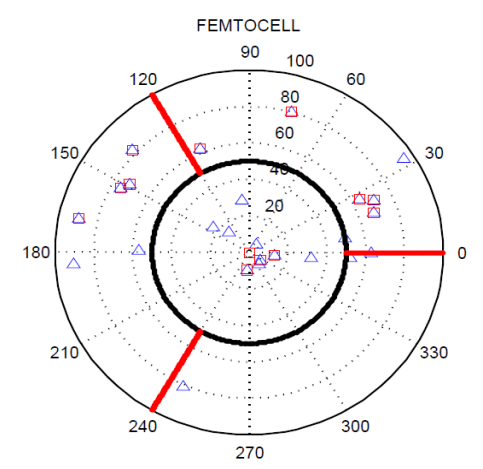

Fig. 2. Movements of femto users in the networks.
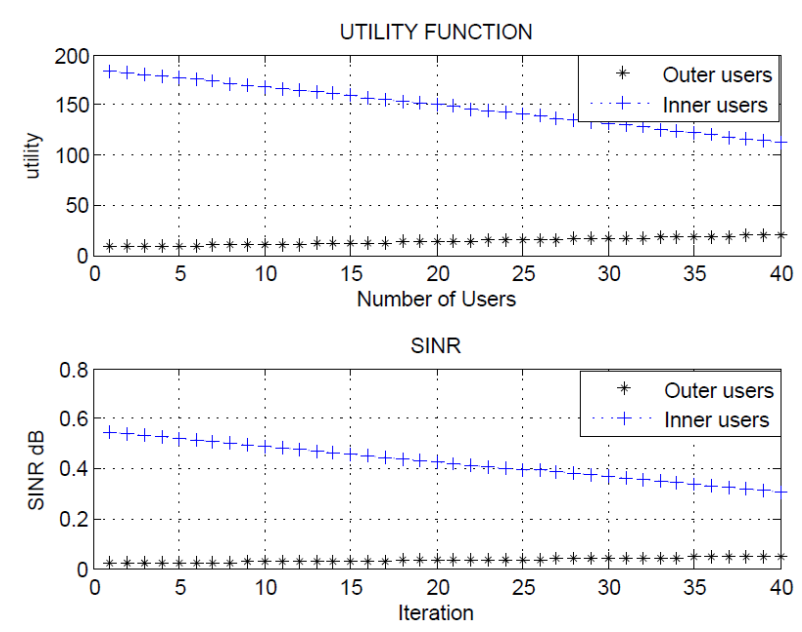

Fig. 3. Utility function of the users and their signal.

Fig. 2 shows us how the femtocell user in each regions of the cell coverage moves around in the cell. Fig. 3 illustrates the utility function of the users at both regions of the cell coverage network, when the utility at the inner user decrease and the utility of at the outer user is constants. At this point, the present of interference still remain un-cancel between the regions due to high power transmitting at inner users at the center region.

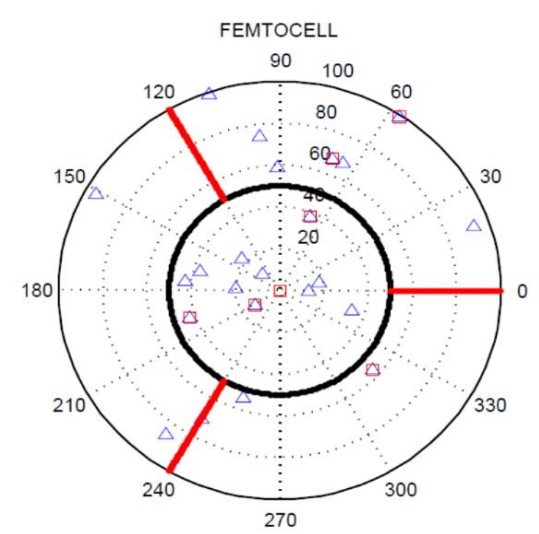

Fig. 4. Show the movements of femto users in the network.
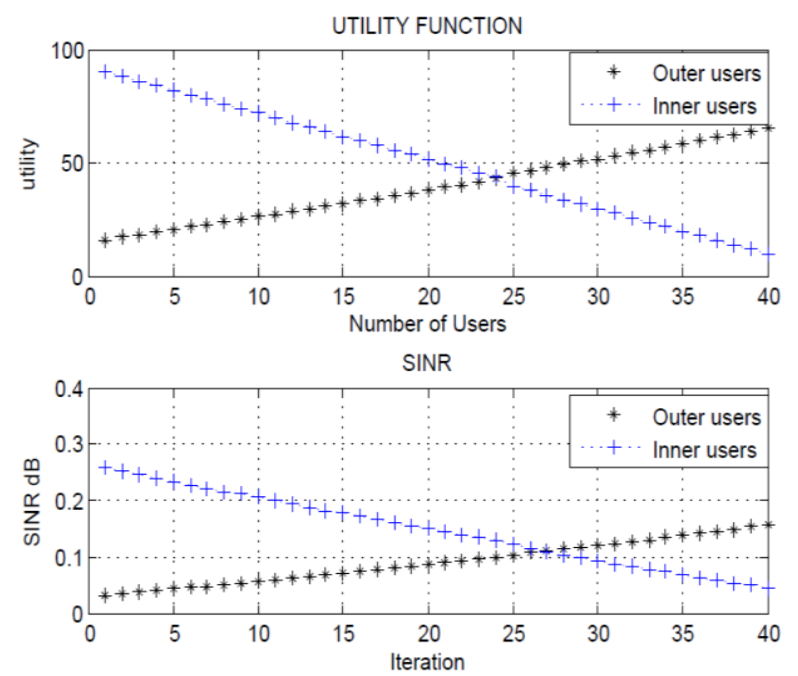

Fig. 5. Nash equilibrium of utility function and signal under number of users.

The Fig. 4 depicts the movement of users in the coverage network. These active users in each region are show here. In Fig. 5, the Nash equilibrium for both the utility function and 
signal is been achieved for the cell network. At this point, interference can be mitigated at the point of Nash equilibrium, where both regions can easily communicate without any interfering to each other. Their received signal power level of transmission are control with their utility functions, in order not to increase their transmitting power level to cause any interference between the regions.

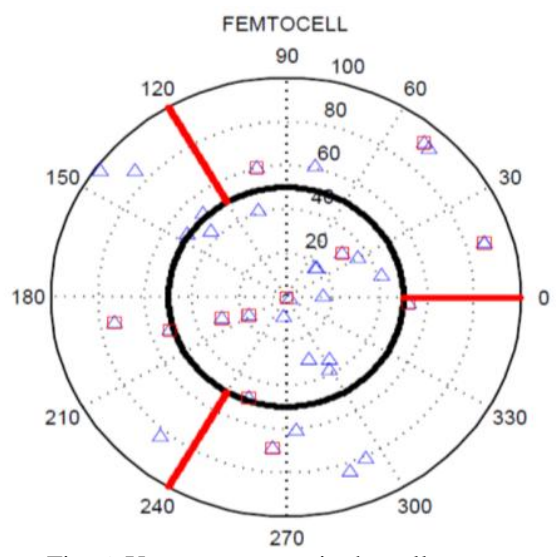

Fig. 6. Users movement in the cell coverage.

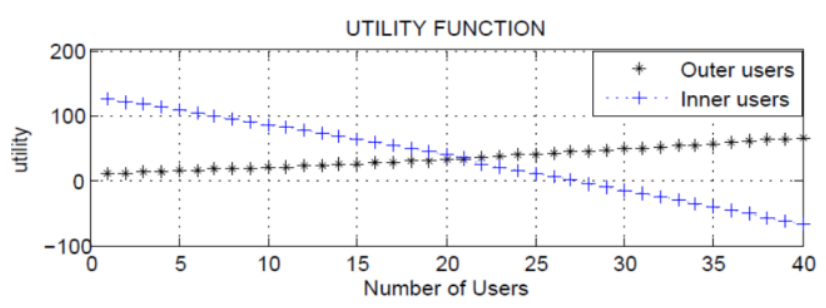

SINR

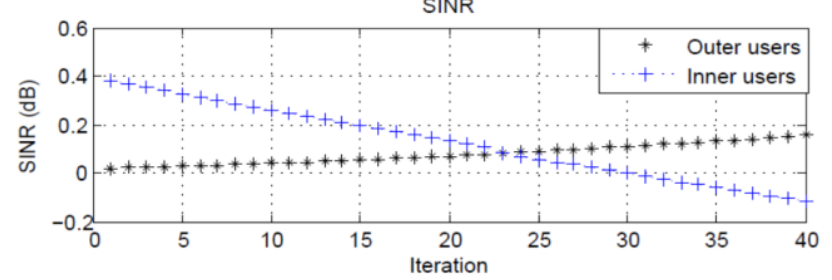

Fig. 7. Nash equilibrium of utility function and signal under number of users.

In Fig. 7, the equilibrium point of the utility function of the regions show that the received signal power at the outer users increase while at the center (inner users) reduced to have the Nash equilibrium and for healthier signal at both regions of the cell networks.

\section{CONCLUSION}

The aim is to mitigate interference in femtocell network between the cell coverage regions where users are located in the cell site coverage. Our technique is based on idea fractional frequency reuse with the concept of price policy, in order to minimize the transmitting power of the femto users at the cell center, so that there will not cause unwanted interference to the whole network. By applying the appropriate definition of utility function, it is possible to sign post the balance between the two regions of the cell coverage area of the networks for quality and capacity performance. This mechanism demonstrates a pure and unique Nash equilibrium as the solution of the formulated problem. With the propose algorithm developed, allow for sharing and protection between the regions.
The price policy brings fairness and quality of service within the regions of the cell site coverage. This proposed scheme is based on mathematically model for cellular system in such a way to minimize the interference in the network.

\section{ACKNOWLEDGMENT}

The authors wish to acknowledge the effort and support of his supervisor and co-supervisors, Dr. Paul B.S and Dr. (Mrs.) Meera K. Joseph of Department of Electrical and Electronic Engineering Technology, University of Johannesburg and Prof. H.C. Ferreira of Deportment of Electrical and Electronic Engineering Science, University of Johannesburg, South Africa.

\section{REFERENCES}

[1] Orthogonal frequency division multiplexing. (August 2013). [Online]. Available: http://www. En.wikipedia.org

[2] 3GPP work items on Self-Organizing Networks. (August 2013). [Online]. Available: http://www.3gpp.org/ftp/Information

[3] T. Alpcan, T. Basar, R. Srikant, and E. Atman, "CDMA uplink power control as a noncooperative game," in Proc. 40th IEEE Conference on Decision and Control, Orlando, FL, December 2001, vol. 1, pp. 197-202.

[4] D. J. Goodman and N. B. Mandayam, "Power control for wireless data," IEEE Personal Communications Magazine, vol. 7, no. 2, pp. 48-54, April 2000.

[5] S. Gunturi and F. Paganini, "A game theoretic approach to power control in cellular CDMA," in Proc. 58th IEEE Vehicular Technology Conference-VTC, Orlando, FL, October 2003, vol. 4, pp. 2362-2366.

[6] C. U. Saraydar, N. B. Mandayam, and D. J. Goodman, "Efficient power control via pricing in wireless data networks," IEEE Transactions on Communications, vol. 50, no. 2, pp. 291-303, February 2002.

[7] A. B. MacKenzie and S. B. Wicker, "Game theory in communications Motivation, explanation, and application to power control," in Proc. IEEE Global Telecommun. Conf., San Antonio, TX, Nov. 2001, pp 821-826.

[8] D. J. Goodman and N. B. Mandayam, "Power control for wireless data," IEEE Pers. Commun., vol. 7, pp. 48-54, Apr. 2000.

[9] H. Ji and C.-Y. Huang, "Non-cooperative uplink power control in cellular radio systems," Wireless Networks, vol. 4, no. 3, pp. 233-240, March 1998.

[10] P. Lee, T. Lee, J. Jeong, and J. Shin, "Interference management in lte femtocell systems using fractional frequency reuse," in Proc. The 12th International Conference on Advanced Communication Technology (ICACT), Feb. 2010, vol. 2, pp. 1047-1051.

[11] H. Lei, L. Zhang, X. Zhang, and D. Yang, "A novel multi-cell OFDMA system structure using fractional frequency reuse," in Proc. IEEE International Symposium on Personal, Indoor and Mobile Radio Communications (PIMRC), Sept. 2007, pp. 1-5.

[12] D. Falomari, N. Mandayam, and D. Goodman, "A new framework for power control in wireless data networks: Games utility and pricing," in Proc. Allerton Conf. on Communication, Control and Computing, Illinois, USA, Sept. 1998, pp. 546-555.

[13] H. Ji and C. Huang, "Non-cooperative uplink power control in cellular radio systems," Wireless Networks, vol. 4, pp. 233-240, March 1998.

Akinlabi Olaniyi Akindeji received the B.Tech degree in electrical and electronic engineering technology in 2007. He is currently working towards his M.Tech degree in electrical and electronic engineering technology at the University of Johannesburg. For academic excellence, he has the best paper Award from IMECS. His research interest focuses in the field of communications and power distribution and generation, interference management, power generation and distribution the major solution to improve both the lack of industrialization and telecommunication infrastructure in remote area.

Meera Joseph has over 10 years' academic and research experience in the information and communication technology (ICT) and social science fields. She engaged with many non-governmental, academic institutions and governmental organizations to ensure rural and urban women's partnership and capacity building in various developmental projects including WiEBE 
Her research interest is "ICT for rural women empowerment". Her passion for web development and her industrial experience in the late 1990's enabled her to design and maintain many web portals for Faculty of Engineering and the Built Environment at UJ including WiEBE for the past few years. Meera Joseph is a senior lecturer at the University of Johannesburg is involved with curriculum development, co-ordination and lecturing IT subjects for the entire faculty.

Hendrik Ferreira received the degrees of B.Sc (Eng.) in electrical (cum laude) in 1976, M.Sc (Eng.) in electronic (cum laude) in 1978, and D.Sc (Eng.) in 1980 from the University of Pretoria. From 1980 to 1981, he was invited to be a post-doctoral researcher at the Linkabit Corporation (predecessor of Qualcomm) in San Diego, CA, USA. In 1983, he joined the
Rand Afrikaans University, Johannesburg, South Africa (currently University of Johannesburg) where he was promoted to professor in 1989 He has served several terms chairing staff and line functions within the RAU Faculty of Engineering's unique pre-1994 matrix structure, and also two terms as Chairman of the Department of Electrical and Electronic Engineering, from 1994 to 1999. Since 1984, he has been a visiting researcher at seven universities and two companies in both the USA and Europe. This includes the Institute for Experimental Mathematics at the University of Duisburg-Essen in Germany, with whom his research group has had a cooperation and exchange agreement since 1992. His research interests are in digital communications and information theory, especially coding techniques. His current applications of the research include power line communications, video communications and networks. 